\title{
MANEJO DEL RIEGO Y ABONADO EN EL CULTIVO DE LA PATATA EN LA COSTA NOROESTE DE CÁDIZ
}

\author{
Salvatierra Bellido, B. (1), Márquez Ruiz, A.J. (1), Luque Sánchez, S.(2), Nieto Martínez, A. \\ (1), Acosta Galán, J.(2). \\ ${ }^{1}$ Instituto de Investigación y Formación Agraria de la Junta de Andalucía (IFAPA). Centro \\ Chipiona, (Cádiz), benito.salvatierra@juntadeandalucia.es \\ ${ }^{2}$ IFAPA. Centro de Jerez, (Cádiz)
}

\section{Resumen}

Desde el Sistema de Asistencia al Regante del Instituto de Investigación y Formación Agraria y Pesquera de Andalucía (IFAPA) en el Centro de Chipiona (Cádiz), se está desarrollando una labor de experimentación de cultivos hortícolas al aire libre. En esta línea se están ensayando cultivos para evaluar la eficiencia del riego y abonado nitrogenado. El objetivo final de estos ensayos es generar unas recomendaciones de riego y fertirrigación útiles para el sector. El cultivo de la patata es un cultivo muy extendido en la zona de Costa Noroeste de Andalucía, por su precocidad en la comarca. El objetivo general del ensayo es determinar de todos los posibles manejos de riego y abonado, cual es la opción más eficiente y más productiva. Para ello se ha utilizado tres dosis de riego, una sobre las necesidades potenciales de agua, otra por encima y otra por debajo, la primera de ellas con manejo dos métodos de riego: aspersión y goteo. Además cada una de estas estrategias con dos dosis distintas de abonado. $\mathrm{Y}$ todo ello replicado en dos parcelas de distintos suelos. El resultado general de todo el abanico de posibilidades ha sido que está muy influenciado del tipo de suelo. Para un suelo más equilibrado el abonado determina mucho más los buenos resultados y con dosis de riego más justas el del abonado es muy determinante. Sin embargo en parcelas de suelo arenoso la dosis de riego en el rendimiento es determinante, pero la variable abonado influye en menor medida en el rendimiento y menos cuando la dosis de riego es más alta, induciéndose a regar con riegos con una mayor frecuencia a la diaria.

\section{1- Introducción y objetivos}

El Centro IFAPA de Chipiona está ubicado en la desembocadura del río Guadalquivir, dónde los cultivos herbáceos al aire libre de regadío son muy variados y dónde desde ahí y hacia el norte (Bajo Guadalquivir) es dónde se aglutina la mayor parte de cultivo herbáceo de regadío de Andalucía. En este centro de Investigación coinciden dos proyectos de experimentación y transferencia del IFAPA relacionados con estos cultivos: el proyecto de Asesoramiento al Regante y el proyecto de cultivos herbáceos al aire libre. El IFAPA tiene entre sus cometidos ejecutar acciones desarrollo experimental que configuren decisiones en la utilización correcta de los fertilizantes, con esta perspectiva se ha trabajado en la asociación entre el manejo de riego y el abonado sobre un cultivo en concreto: la patata. El cultivo de la patata es un cultivo muy extendido en la zona de Costa Noroeste por la precocidad que ofrece el clima en esta zona. En el mercado nacional los precios son muy oscilantes y requieren de una mayor profesionalización del sector. El objetivo ha sido determinar cuál es el mejor manejo para optimizar los recursos agua y abonado. Ambos factores hoy en día también son determinantes para la rentabilidad del cultivo. Igualmente es 
necesario estudiar su interacción para afinar en el manejo de agua condicionado por otro recurso que cada vez es más caro y más controlado, el nitrógeno en el suelo.

Además del objetivo general de determinar la opción más eficiente y más productiva utilizando las variables de dosis de abonado y dosis de riego sobre el cultivo de la patata, se integran los siguientes objetivos específicos:

- Ajuste del coeficiente de cultivo $(\mathrm{Kc})$ para nuestra zona y por tanto la recomendación del riego.

- Ajuste de recomendación de abonado para maximizar producción y la calidad del producto.

- Comparación entre el sistema de riego localizado y por aspersión en el cultivo de la patata.

- Interacción entre el manejo del riego y el abonado en este cultivo.

El propósito final de estos ensayos es generar unas recomendaciones de riego y fertirrigación útiles para el sector.

\section{2- Materiales y métodos}

Para los objetivos propuestos se han utilizado dos parcelas experimentales muy representativas de los dos tipos de suelos utilizados para el cultivo de la patata en la zona. Se denominará a las parcelas utilizadas: Parcela 1 o de Jerez de la Frontera y Parcela 2 o de Chipiona. Para el diseño hemos contados con riego localizado en ambas parcelas y en la Parcela 2 además de riego localizado se cuenta con riego por aspersión. El sistema de riego por aspersión es el que mayoritariamente utilizan los agricultores de la comarca de Costa Noroeste.

La localización exacta de ambas se expone en la siguiente tabla:

Tabla 1. Datos de localización la Parcela 1

\begin{tabular}{|l|c|c|}
\hline $\begin{array}{l}\text { Localizaciones } \\
\text { de las parcelas }\end{array}$ & Parcela 1 & Parcela 2 \\
\hline Provincia & 11 (Cádiz) & 11 (Cádiz) \\
Municipio & 20 (Jerez de la Frontera) & 16 (Chipiona) \\
Polígono & 106 & 14 \\
Parcela & 18 & 76 \\
Superficie & $2050 \mathrm{~m}^{2}$ & $1774 \mathrm{~m}^{2}$ \\
\hline
\end{tabular}

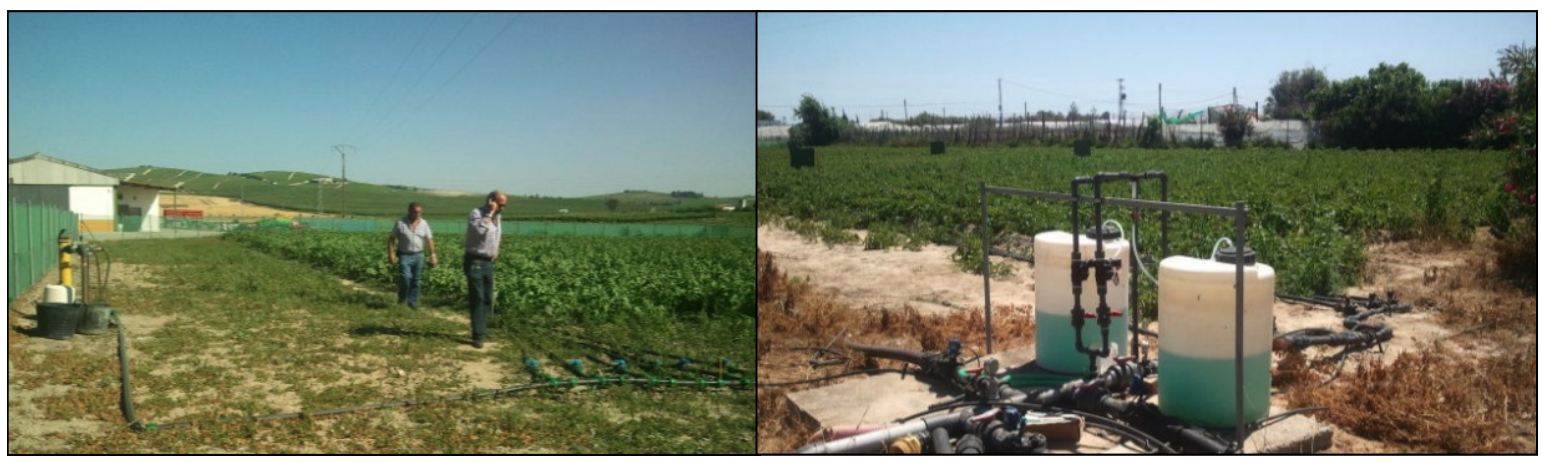

Figura 1. Imágenes del desarrollo de los ensayos de las dos parcela. La 1 a la izquierda y la 2 a la derecha. 
Las diferencias físicas de ambas parcelas son las siguientes-

\section{Caracteristicas del suelo:}

- Parcela 1: Presenta una textura franco arcillosa. Con una conductividad eléctrica de $1,4 \mathrm{mS} / \mathrm{cm}$ en el extracto saturado, quedando muy por debajo del límite para considerarlo salino en $4 \mathrm{mS} / \mathrm{cm}$ y un nivel bajo de sodio, situado en $0,44 \mathrm{meq} / 100 \mathrm{gr}$.

- Parcela 2 presenta una textura arenosa (90\% arena), con un nivel de materia orgánica de $1,09 \%$, el cual podemos considerar como un nivel medio-bajo. La conductividad eléctrica nos indica la salinidad del suelo, $3,15 \mathrm{mS} / \mathrm{cm}$ en el extracto saturado lo cual hace que el suelo sea ligeramente salino, quedando el límite para considerarlo salino en $4 \mathrm{mS} / \mathrm{cm}$, y es un factor a tenido en cuenta en el ensayo.

\section{Características del agua:}

- Parcela 1: El agua utilizada este ensayo proviene de un pozo con una conductividad eléctrica en de $1,98 \mathrm{dS} / \mathrm{m}$

- Parcela 2: Esta agua es proporcionada por la comunidad de regantes. Tiene valores oscilantes de conductividad eléctrica, pero con un valor máximo al final del cultivo de de $3.09 \mathrm{mS} / \mathrm{cm}$ a $25^{\circ} \mathrm{C}$, con lo que se ha hecho un manejo de riego con las correspondientes necesidades de lavado de cada momento y los resultados esperados tendrán un nivel menor de producción.

\section{Material vegetal:}

Para el ensayo se utilizo la variedad "Spunta" en ambas parcelas. Esta esta variedad está clasificada como semitemprana debido a que su ciclo de cultivo tiene una duración de unos 120 días aproximadamente.

\section{Material de riego:}

En ambas parcelas el sistema de riego por goteo es similar, en la parcela 2 además tiene un tratamiento más de riego por aspersión incluido en el diseño experimental y dispone de las siguiente caracterísiticas

- Goteros: $4 \mathrm{l} / \mathrm{h}$; Marco 0,33 x 0,8 metros.

○ Laterales: diámetro de $16 \mathrm{~mm}$., $4 \mathrm{~atm}$.

- Portalaterales: diámetro de $40 \mathrm{~mm}$., $6 \mathrm{~atm}$.

\section{- Aspersion:}

- Aspersores: $1092 \mathrm{l} / \mathrm{h}$, boquilla $5 / 32+3 / 32$, marco $12 \times 12 \mathrm{~m}$., todos sectoriales de $180^{\circ}$, pluviometría de $15,1 \mathrm{l} / \mathrm{m}^{2}$ hora.

\section{Diseño experimental:}

En ambas parcelas se planteó un experimento sobre el efecto de las variables de riego y de fertilización nitrogenada en el cultivo de la patata. Se ensayaron tres niveles de riego y dos niveles de abonado variando sólo el nivel de nitrógeno como se detallan en las tablas siguientes. 
Tabla 1. Tratamientos de riego

\begin{tabular}{|l|l|l|l|}
\hline R1 & R2 & R3 & R4 \\
\hline Riego localizado & Riego localizado & Riego localizado & Riego por \\
$80 \%$ de la dosis & $\begin{array}{l}100 \% \text { de la dosis } \\
\text { potencial }\end{array}$ & $\begin{array}{l}120 \% \text { de la dosis } \\
\text { potencial }\end{array}$ & $\begin{array}{l}\text { aspersión 100\% de } \\
\text { la dosis potencial }\end{array}$ \\
\hline
\end{tabular}

Tabla 2. Tratamientos de abonando

\begin{tabular}{|l|c|c|}
\hline Unidades de fertilizantes & Dosis de abonado 1. (A1) & Dosis de abonado 2. (A2) \\
\hline Nitrógeno & 189 & 261 \\
\hline Fósforo & 150 & 150 \\
\hline Potasio & 300 & 300 \\
\hline
\end{tabular}

De cada abonado de fertirrigación se fijaron los siguientes planificaciones de dosis (Oltra et al., 2006):

Tabla 3. Distribución del abonado 1

\begin{tabular}{|l|c|ccc|}
\hline \multicolumn{2}{|l}{$\begin{array}{l}\text { ABONADO 1 (A1) } \\
\text { TOTAL }\end{array}$} & $\begin{array}{c}\mathbf{N} \\
\mathbf{1 8 8}\end{array}$ & $\begin{array}{c}\mathbf{P}_{\mathbf{2}} \mathbf{O}_{\mathbf{5}} \\
\mathbf{1 4 0}\end{array}$ & $\begin{array}{c}\mathbf{K}_{\mathbf{2}} \mathbf{O} \\
\mathbf{3 0 1}\end{array}$ \\
\hline FONDO & Semana & $\mathrm{Kg} / \mathrm{ha}$ & $\mathrm{Kg} / \mathrm{ha}$ & $\mathrm{Kg} / \mathrm{ha}$ \\
\hline & $1-5$ & 22,5 & 30,0 & 0,0 \\
\hline Emergencia & $6-7$ & 7,6 & 10,0 & 0,0 \\
\hline Estolonización & $8-10$ & 63,6 & 0,0 & 63,9 \\
\hline $\begin{array}{l}\text { Comienzo de la } \\
\text { tubérculo }\end{array}$ & $11-13$ & 33,9 & 0,0 & 63,9 \\
\hline \multirow{2}{*}{ Relleno del tubérculo } & $14-16$ & 0 & 0,0 & 20,1 \\
\hline
\end{tabular}

Tabla 4. Distribución del abonado 2

\begin{tabular}{|c|c|c|c|c|}
\hline $\begin{array}{l}\text { ABONADO } 2 \text { (A2) } \\
\text { TOTAL }\end{array}$ & & $\begin{array}{c}N \\
260 \\
\end{array}$ & $\begin{array}{c}\mathrm{P}_{2} \mathrm{O}_{5} \\
140 \\
\end{array}$ & $\begin{array}{l}\mathrm{K}_{2} \mathrm{O} \\
301 \\
\end{array}$ \\
\hline \multirow[t]{2}{*}{ FONDO } & & 83,5 & 100,0 & 153,3 \\
\hline & Semana & $\mathrm{Kg} / \mathrm{ha}$ & $\mathrm{Kg} / \mathrm{ha}$ & $\mathrm{Kg} / \mathrm{ha}$ \\
\hline Emergencia & $1-5$ & 31,5 & 30,0 & 0,0 \\
\hline Estolonización & $6-7$ & 10,4 & 10,0 & 0,0 \\
\hline $\begin{array}{l}\text { Comienzo de la } \\
\text { tubérculo }\end{array}$ & 8-10 & 87,6 & 0,0 & 63,9 \\
\hline \multirow{2}{*}{ Relleno del tubérculo } & $11-13$ & 47,1 & 0,0 & 63,9 \\
\hline & $14-16$ & 0,0 & 0,0 & 20,1 \\
\hline
\end{tabular}

La unidad experimental fueron parcelas con tres líneas de cultivo en un diseño de bloques completos al azar. El experimento fue un diseño de tipo factorial con las variables dosis de riego y dosis de abonado.e incluye tres repeticiones, ( seis combinaciones posibles en cada bloque) como se puede observar en la figura 2.En el diseño de los ensayos se incluye entre cada tratamiento de riego una línea borde sin cultivar. 

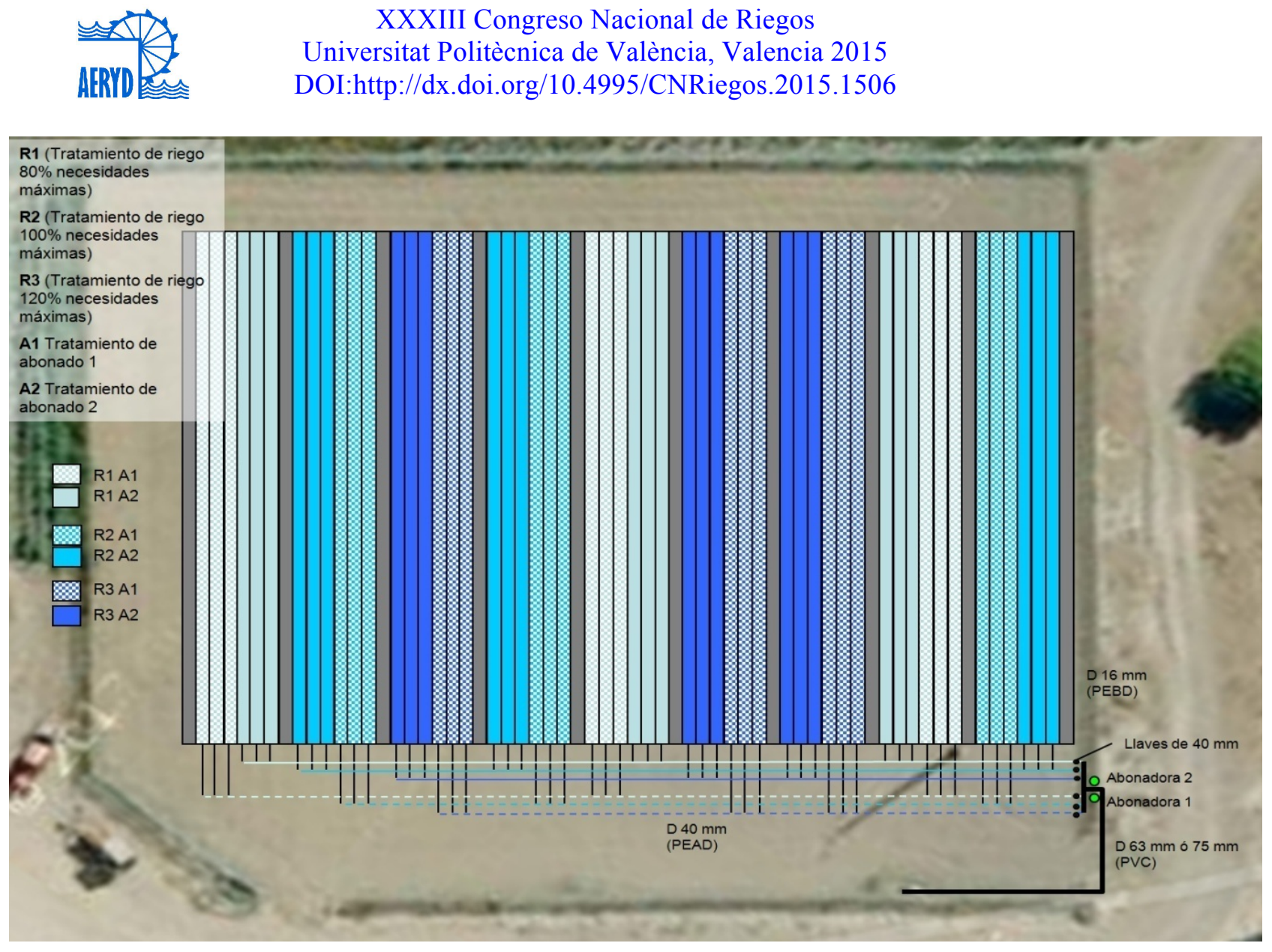

Figura 2. Esquema estándar del diseño experimental del riego localizado de las dos parcelas.

En la parcela 1 existe un cuarto tratamiento, para ensayar una dosis del $100 \%$ a través de riego por aspersión dos los dos tratamientos de abonado $(\mathrm{A} 1, \mathrm{~A} 2)$ pero mediante la aplicación de dos coberteras de abono sólido convencional. El objetivo de este cuarto tratamiento es hacer constar el manejo tradicional de riego y abonado de la comarca para comparar las ventajas de eficiencia de riego y abonado y de producción del riego localizado con fertirrigación frente a el manejo tradicional. Se ha realizado en la parcela 1 por contar con un suelo de textura arenosa, dónde existe un mayor riesgo de lavado de Nitrógeno y pérdidas de agua por percolación. En este caso se resaltarían, dentro del mismo ensayo, las diferencias productivas entre ambos manejos con mayor claridad.

\section{Metodología de evaluación del ensayo:}

Los valores a medir en estos ensayos fueron el rendimiento del cultivo y su calibre. Se recolectó la línea central de cada repetición, cada una fue pesada y calibrada según los siguientes rangos de calibre: menor de $40 \mathrm{~mm}$ de diámetro, entre 40 y $60 \mathrm{~mm}$, entre 60 y 80 $\mathrm{mm}$ y mayor de $80 \mathrm{~mm}$.

\section{Analisis estadístico:}

Con los distintos datos de producción recogidos, a diferentes niveles de abonado de riego, se ha realizado el análisis de varianzas Se realizo un análisis de varianza de diseño factorial teniendo usando las variables riego y abonado con una disposición en bloque (en nuestro caso tenemos tres bloques de repetición en cada ensayo). 


\section{3- Resultados y discusión}

Los principales datos obtenidos son los resultados de producción, de calidad de la patata y de seguimiento del riego de cada estrategia de riego. A continuación se detallan cada bloque de datos.

Los datos de producción de cada repetición de los tratamientos. Cada repetición viene designada por el número del tratamiento de riego " $R$ " y el número del tratamiento de abonado "A"

Tabla 5. Datos de producción (Kg/ha) pertenecientes a la parcela 1

\begin{tabular}{|c|c|c|}
\hline Bloque & Tratamientos & $\mathrm{Kg} / \mathrm{ha}$ \\
\hline 1 & $\mathrm{R} 2 \mathrm{~A} 1$ & 50.854 \\
1 & $\mathrm{R} 2 \mathrm{~A} 2$ & 48.811 \\
1 & $\mathrm{R} 1 \mathrm{~A} 2$ & 48.933 \\
1 & $\mathrm{R} 1 \mathrm{~A} 1$ & 52.713 \\
1 & $\mathrm{R} 3 \mathrm{~A} 2$ & 46.616 \\
1 & $\mathrm{R} 3 \mathrm{~A} 1$ & 54.878 \\
\hline 2 & $\mathrm{R} 3 \mathrm{~A} 2$ & 46.250 \\
2 & $\mathrm{R} 3 \mathrm{~A} 1$ & 52.043 \\
2 & $\mathrm{R} 1 \mathrm{~A} 1$ & 52.409 \\
2 & $\mathrm{R} 1 \mathrm{~A} 2$ & 41.585 \\
2 & $\mathrm{R} 2 \mathrm{~A} 2$ & 40.915 \\
2 & $\mathrm{R} 2 \mathrm{~A} 1$ & 50.793 \\
\hline 3 & $\mathrm{R} 3 \mathrm{~A} 2$ & 41.951 \\
3 & $\mathrm{R} 3 \mathrm{~A} 1$ & 47.012 \\
3 & $\mathrm{R} 2 \mathrm{~A} 2$ & 35.884 \\
3 & $\mathrm{R} 2 \mathrm{~A} 1$ & 48.659 \\
3 & $\mathrm{R} 1 \mathrm{~A} 1$ & 51.646 \\
3 & $\mathrm{R} 1 \mathrm{~A} 2$ & 46.463 \\
\hline
\end{tabular}

De los datos anteriores nos interesan los calibres comprendidos entre 40 y $80 \mathrm{~mm}$ por ser los más comercializables. Estos calibres representan un porcentaje de la producción total (tabla 6 y 8 ) y puede ser uno de los valores que se ven afectados tanto por el riego como por el abonado.

Tabla 6. Porcentajes de calibre comerciales cosechados en la parcela 1.

\begin{tabular}{|c|c|c|c|c|c|c|}
\hline \multicolumn{7}{|c|}{ Porcentajes de patata con calibres entre 40 y 80 mm de la parcela 1} \\
\hline BLOQUE & \multicolumn{7}{|c|}{ PARCELAS EXPERIMENTALES } \\
\hline \multirow{2}{*}{1} & R2A1 & R2A2 & R1A2 & R1A1 & R3A2 & R3A1 \\
\cline { 2 - 7 } & 59,66 & 84,55 & 66,14 & 76,45 & 78,47 & 67,30 \\
\hline \multirow{2}{*}{2} & R3A2 & R3A1 & R1A1 & R1A2 & R2A2 & R2A1 \\
\cline { 2 - 7 } & 85,66 & 84,82 & 75,79 & 90,00 & 89,75 & 78,82 \\
\hline \multirow{2}{*}{3} & R3A2 & R3A1 & R2A2 & R2A1 & R1A1 & R1A2 \\
\cline { 2 - 7 } & 89,22 & 95,62 & 87,64 & 91,44 & 78,03 & 86,79 \\
\hline
\end{tabular}


Tabla 7. Datos de producción $(\mathrm{Kg} / \mathrm{ha})$ pertenecientes a la parcela 1

\begin{tabular}{|c|c|c|c|}
\hline Parcela & Bloque & Tratamientos & Kg/ha \\
\hline 1 & 1 & R1A2 & 26923,1 \\
2 & 1 & R1A1 & 27243,6 \\
3 & 1 & R2A2 & 30288,5 \\
4 & 1 & R2A1 & 24519,2 \\
5 & 1 & R3A2 & 31250,0 \\
6 & 1 & R3A1 & 29647,4 \\
\hline 7 & 2 & R2A2 & 27604,2 \\
8 & 2 & R2A1 & 19791,7 \\
9 & 2 & R3A2 & 35590,3 \\
10 & 2 & R3A1 & 37152,8 \\
11 & 2 & R1A2 & 27256,9 \\
12 & 2 & R1A1 & 22916,7 \\
\hline 13 & 3 & R1A2 & 24905,3 \\
14 & 3 & R1A1 & 19412,9 \\
15 & 3 & R3A2 & 28409,1 \\
16 & 3 & R3A1 & 29545,5 \\
17 & 3 & R2A2 & 25947,0 \\
18 & 3 & R2A1 & 20075,8 \\
\hline
\end{tabular}

Tabla 8. Porcentajes de calibre comerciales cosechados en la parcela 2.

\begin{tabular}{|c|c|c|c|c|c|c|}
\hline \multicolumn{7}{|c|}{ Porcentajes de patata con calibres entre 40 y $80 \mathrm{~mm}$ de la parcela 2} \\
\hline \multirow{2}{*}{1} & RLOQUE & \multicolumn{7}{|c|}{ PARCLAS EXPERIMENTALES } \\
\cline { 2 - 7 } & 88,99 & R1A1 & R2A2 & R2A1 & R3A2 & R3A1 \\
\hline \multirow{2}{*}{2} & R2A2 & R2A1 & R3A2 & R3A1 & R1A2 & R1A1 \\
\cline { 2 - 7 } & 91,72 & 92,64 & 97,77 & 98,89 & 95,37 & 95,18 \\
\hline \multirow{2}{*}{3} & R1A2 & R1A1 & R3A2 & R3A1 & R2A2 & R2A1 \\
\cline { 2 - 7 } & 93,63 & 94,92 & 94,67 & 83,33 & 95,11 & 94,83 \\
\hline
\end{tabular}

El manejo del riego fue programado semanalmente en función del método de balance de agua en el suelo (Allen et al., 1998), utilizando sendas estaciones agroclimáticas de la Red de Información Agroclimática de Andalucía. Se aplicaron dosis medias semanales en función a la dosis indicada para cada estrategia de riego. El resultado de la evolución del Déficit de Agua en el Suelo (DAS) para cada parcela y durante la campaña de cada estrategia, se representa en las figuras 4 y 5 . En estas también se fija los valores de Nivel de Agotamiento Permisible para el cultivo de la patata según Allen et al., 1998. El punto de marchitez permanente del cultivo se representa como valor inferior de Intervalo de Humedad Disponible (IHD). 


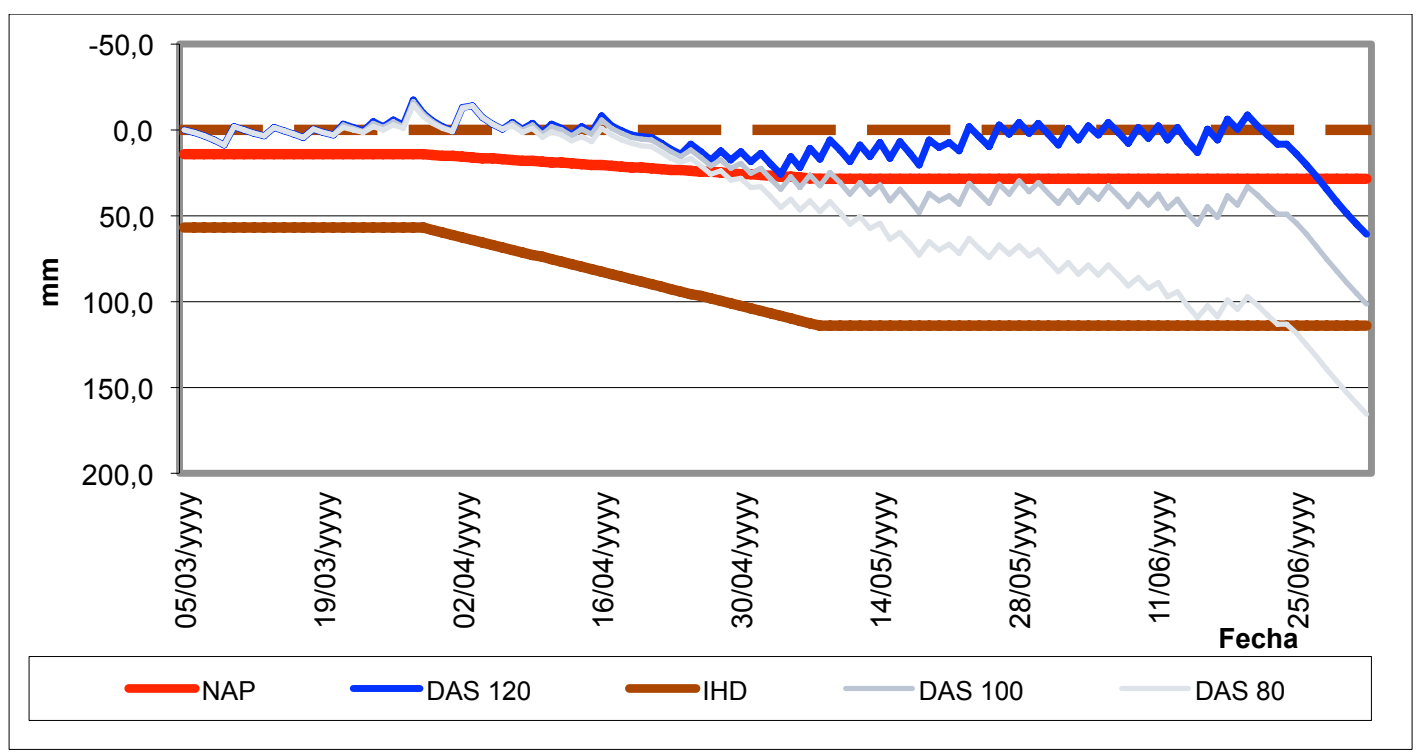

Figura 4 Gráfico de la evolución de los Déficit de Agua en el Suelo (DAS) para los distintos tratamientos de riego de la parcela 1.

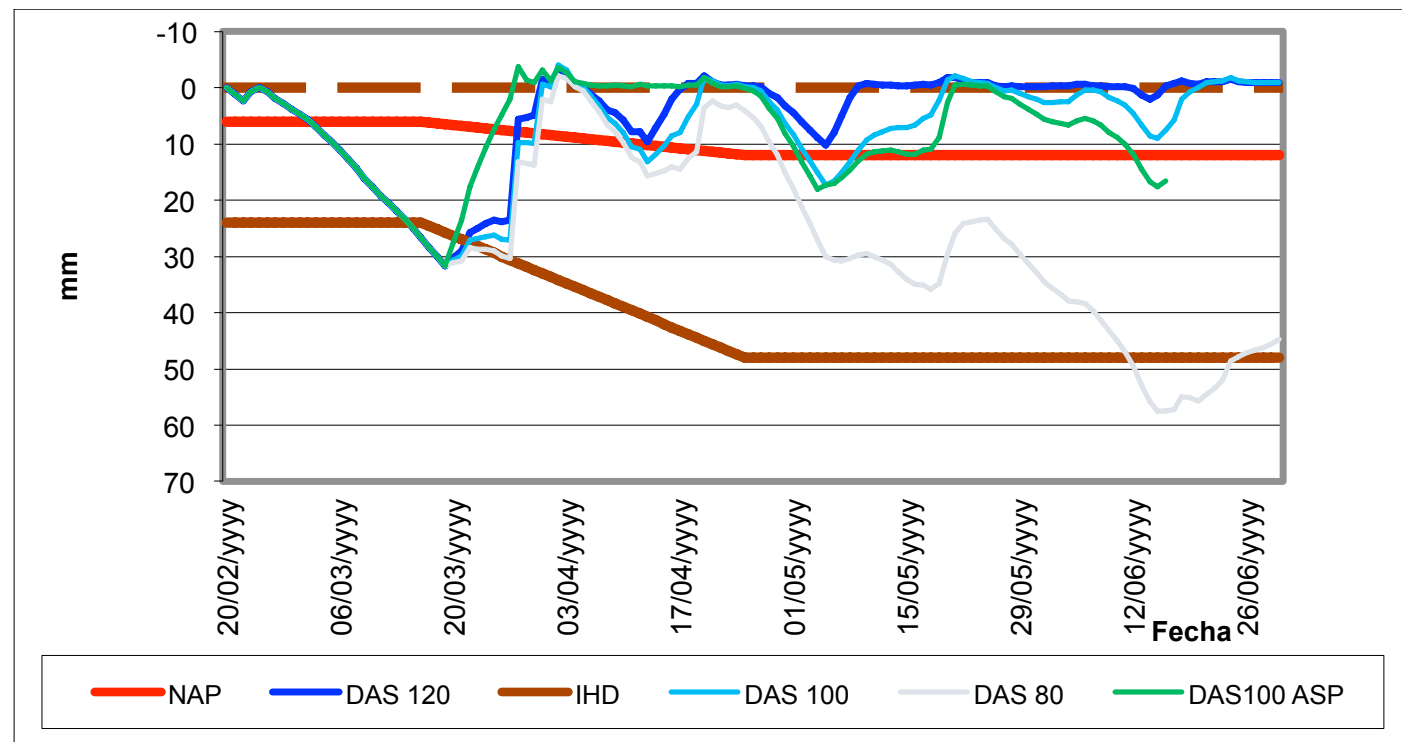

Figura 5 Gráfico de la evolución de los Déficit de Agua en el Suelo (DAS) para los distintos tratamientos de riego de la parcela 2 .

Como resumen de ambas parcelas en el habito de riego, cabe destacar la regularidad de los episodios de riego en la parcela 1 frente a la parcela 2, pudiendo éste además de la salinidad el factor que baje el nivel productivo de la parcela 2 frente a la 1.

En la parcela de aspersión se refleja un peor ajuste a la parcela testigo (dosis del $100 \%$ de goteo) pero situada entre la dosis superior e inferior. Con los datos expuestos se realiza un análisis de varianza de diseño factorial, usando las variables riego y abonado y con disposición en bloque. Este análisis se realiza tanto para rendimiento como para calibre de calidad. El fin de este análisis es comprobar de forma fehaciente la influencia de las distintas variables en la productividad. 
Para una probabilidad de 0.05 , se observa una clara influencia de la variable abonado en el rendimiento, en cambio la variable riego en nuestro caso tiene una influencia mucho menor (no significativa). La combinación de abono y riego arroja un valor mucho mayor y por tanto se deduce que su influencia es no significativa.

A continuación en las figuras 6 y 7 se representan los valores de producción en relación con el abonado y dosis de riego.

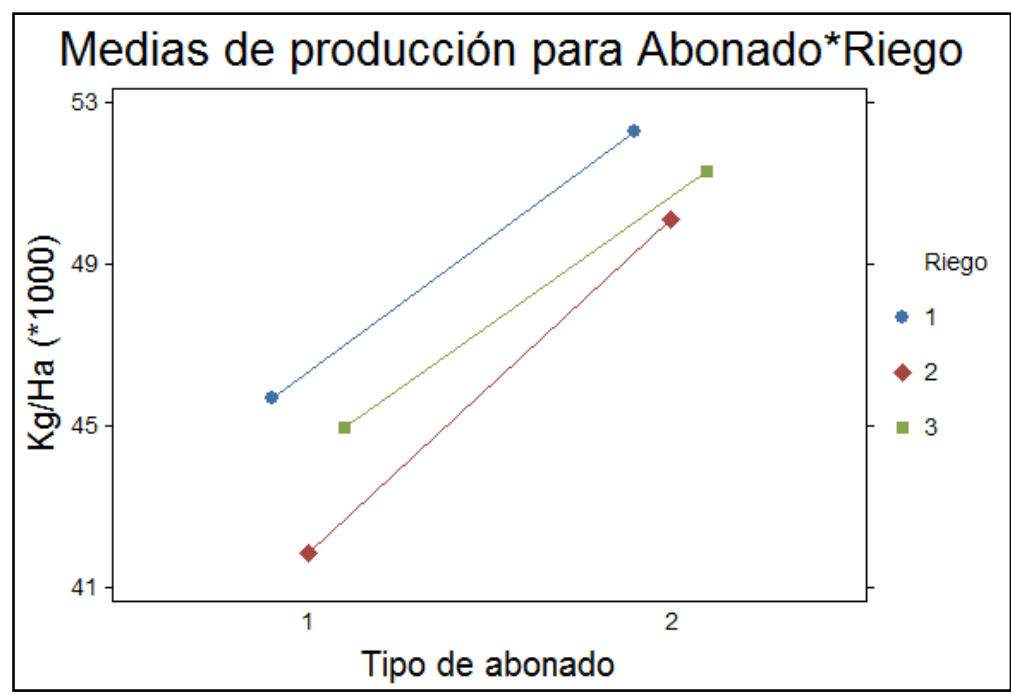

Figura 6 Gráfico de medias de producción para la parcela 1 diferenciadas para las dos estrategias de abonada y diferenciadas por las dosis de riego $(1,120 \% ; 2,100 \%$ y $3,80 \%)$.

En el gráfico de medias anterior se ve claramente la total influencia del abonado en la producción, mientras el riego es poco o nada determinante (las líneas tienen una disposición casi paralela)

En la parcela 2 existe una clara influencia de la variable "riego" en el rendimiento, además con suficiente significación. En menor medida, la variable abonado influye en el rendimiento.

También se observa que la combinación de riego y abonado no aporta la suficiente significación para ser tenida en cuenta, por tanto abonado y riego serán tenidos en cuenta de forma separada.

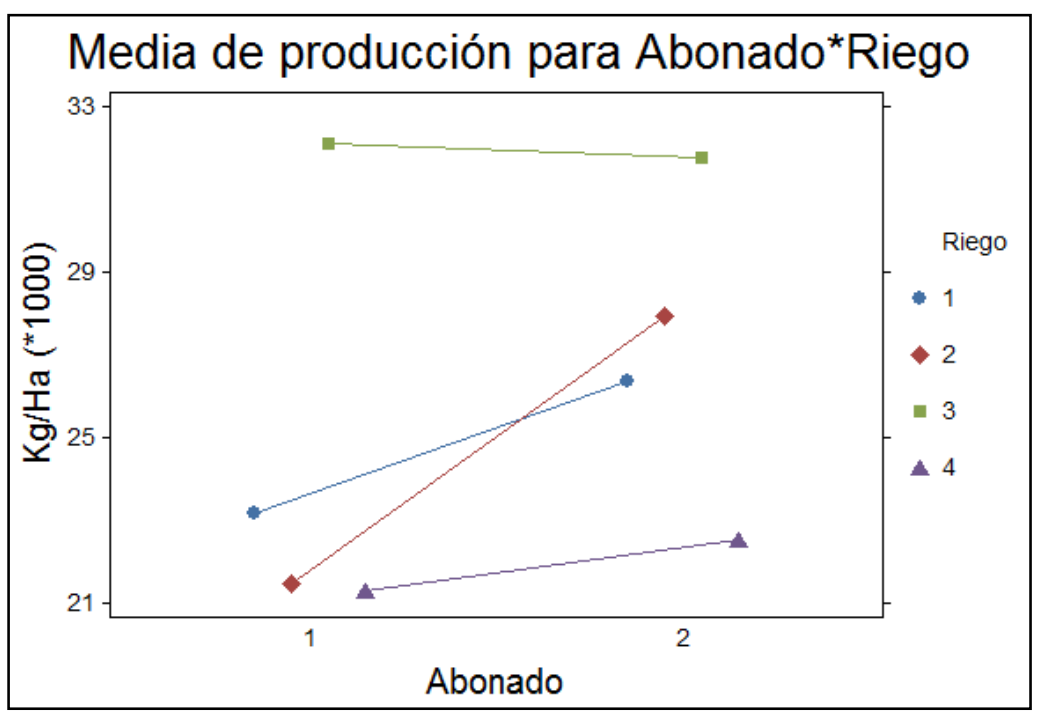


Figura 7 Gráfico de medias de producción para la parcela 2.

En la figura anterior se ve claramente la influencia del abonado en la producción, mientras el riego donde existe una total diferencia es en el tipo 3 (120\% de las necesidades)

\section{4- Conclusiones y recomendaciones}

Los datos obtenidos ratifican las ventajas del sistema de riego localizado y la fertirrigación para este cultivo, con las siguientes consideraciones:

Para la parcela 1 con el suelo franco arcilloso se observa lo siguiente:

- Una clara influencia de la variable abonado sobre el rendimiento, en relación a la significación de la variable dosis riego.

- El aumento del abonado nitrogenado influye de forma determinante en el aumento de producción en las tres dosis de riego, siendo la dosis del $100 \%$ de las necesidades, la que mejor responde a este aumento de nitrógeno.

- En la dosis de riego del $80 \%$ de las necesidades existe una media de producción más alta pero no significativa para ambos niveles de abonado. Por tanto podemos ir a una dosis ligeramente más baja en la programación de riego.

Para la parcela 2 con el suelo arenoso, se ha obtenido las siguientes conclusiones:

- Una influencia determinante de la dosis de riego en el rendimiento, además con suficiente significación, pero en menor medida, la variable abonado influye en el rendimiento y menos cuando la dosis de riego es más alta.

- Queda claramente diferenciado que el riego de $120 \%$ produce un mayor rendimiento y esto puede ser explicado por diversas razones:

- Por el tipo de suelo (90\% de arena), con poca de retención. Por tanto al ser un riego localizado, el mayor tiempo de riego implica un mayor volumen de humectación y un mayor tiempo de riego. No teniendo en este caso excesiva influencia el abonado por realizarse siempre al final del riego.

- La calidad del agua, esta tiene una conductividad eléctrica de 3,09 mS/cm, valor bastante elevado que aun habiendo hecho el precedente lavado, provoca una ligera barrera en la absorción de agua y nutrientes.

- El tratamiento de riego por aspersión con el 100\% de la dosis, obtiene el menor rendimiento medio, esto se debe a la menor uniformidad en la aplicación de riego y al método de aplicación del abono (aplicación de fondo y dos coberteras). . Otra causa es el estrés que provoca del agua de riego en la parte aérea de la planta, produciéndose una daño foliar y fitotoxicidad.

- Para un riego del $100 \%$ en riego localizado, existe una mayor respuesta al abonado que en el resto de los riegos.

Las producciones más altas de la parcela 1 con respecto a la parcela 2 demuestran que es necesario regar la parcela 2 con más de un ciclo de riego diario, tanto por el suelo, como por el posible efecto de la salinidad.

\section{5- Bibliografía}

Allen, R.G., Pereira, L.S., Raes and D., Smith, M. (1998). "Crop evapotranspiration: Guidelines for computing crop water requirements". FAO Irrigation and Drainage Paper No 56 , Rome.

Alonso Arce, Fernando. El cultivo de la patata. Madrid: Mundi-Prensa, 2002. 
Oltra Cámara, Marco Antonio, et al. "Óptima fertilización para un alto rendimiento de patata (Solanum tuberosum L.)". En: Nutrición mineral : aspectos fisiológicos, agronómicos y ambientales / Carmen Lamsfus Arrien (editora-coordinadora) ; Pedro M. Aparicio Tejo...[et al.] (editores). Pamplona : Universidad Pública de Navarra, 2006. Vol. 2. ISBN 84-9769-1644, pp. 375-381 